\title{
Android Cellular Phone Instant-Run off Ballot Systems
}

\author{
G.Suseendran \\ Assistant Professor \\ Department of Information Technology \\ Vels University, Chennai \\ suseendar_1234@yahoo.co.in \\ Sreehari V Kumar, \\ Dept of MSc IT, \\ Vels University, Chennai
}

\begin{abstract}
This venture is entitled "Portable voting framework" is produced by utilizing android as front end and SQLite as Back End. Amid decision period the rate of making of choice is turning out to be less step by step. The Indian subjects the individuals who are living in different nations can't make their choice, those whose are debilitated, old matured and for all intents and purposes disable. Every last individual has their own particular appropriate to vote as an Indian native and it is additionally a control followed in the nation. We give a chance to the general population the individuals who are not ready to vote. Our application depends both on security and coordinated web administrations to vote from anyplace with every single vital evidence, for example, voter id, photograph, Aadhar card, and so forth. An occasion can be depicted as an open get together with the end goal of festivity, instruction, showcasing, get-together or to settle on a choice. It as a rule requires a lot of investment and exertion from many individuals to put on an occasion of significance. Sometimes is difficult to settle on a choice on whether to offer for, and after that compose, a noteworthy occasion. Individuals will have varying perspectives dependant regularly on how agreeable they are with the degree of work that is included. The center of the application is to broaden the usefulness of the cell phone which can be utilized as opposed to voting frameworks' remote parts. The fundamental objective of the application in view of customer server design is to wipe out the overhead related with the remote parts taking care of. The effects of the application are decrease in time; voting number may increment up to $20 \%$ to $30 \%$ than the present situation, bringing about less issues in discretionary arrangements, peace, hopeful's use and give simple and precise checking with no devilishness at the tallying focus.
\end{abstract}

Keywords: Voting, Mobile Voting, Voting Security, Android.

\section{INTRODUCTION}

In web based voting system a voter can utilize hislher voting right online with no trouble. HelShe must be enrolled first for him/her to vote. Enlistment is primarily done by the framework chairman for security reasons. The framework Administrator enrolls the voters on an extraordinary site of the framework went to by him just by essentially filling an enlistment shape to enlist voter. Natives looking for enrollment are required to contact the framework manager to present their points of interest. [1][6][7]New System Of E-Voting Using Fingerprint: One of the most seasoned techniques utilized as a part of various applications. After the legitimacy of them being subjects of India has been affirmed by the framework overseer by contrasting their points 
of interest submitted and those in existing databases, for example, those as the Registrar of Persons, the national is then enrolled as a voter.

The proposed arrangements were correspondingly plot to keep down these assaults. For instance, to maintain a strategic distance from programmer making invasion into the voting framework by means of system, we can plan our framework to transmit information without system [3][8].Next Generation A Secure E-Voting System Based on Biometric Fingerprint Method: Because of fast development of innovation security issues are getting expanded. Another illustration is to constrain voter to info specific information, with the goal that we can keep the order infusion from running.

The correct execution of fair rights has turned out to be connected to the accessibility and solid working of cutting edge data and correspondence innovation (ICT) [2].Qr Codes and Security Solutions: QR Code is a Matrix code; the QR codes were created in Japan in 1994 by Toyota backup, Denso Wave to help track vehicle parts all through generation. While current social orders completely depend on ICT for business, work and relaxation time exercises, the utilization of ICT for law based basic leadership is still in its earliest stages[5][10]. Design A Secure Electronic Voting System Using Fingerprint Technique: Unique finger impression biometric is the most broadly sent plugged biometrics for distinguishing proof.. Iris acknowledgment can't occur without your consent. Actually, the out date innovative ideas for voting have been rebuked to some extent for lost and uncounted votes and could hence is in charge of one-sided political choices making [4].A Novel Data Hiding Technique Based BioSecure Online Voting System: Electronic voting is an incredible change over paper frameworks Nations everywhere throughout the world are inspecting e-voting, for it makes them strike favorable circumstances over customary paper voting, including security for throwing votes, exactness of tallying and investigating votes, choices to lead voting in a brought together and decentralized way, and so on. The reasons why the e-voting innovation has not developed to equal levels as known for business and recreation time exercises lies for the most part in a characteristic absence of trust and dread of electronic dangers. While most nations are as yet conceptualizing or testing e-voting frameworks, three cantons in Switzerland have spearheaded the improvement of e-voting to its full mechanical development. The world is dependably in change and development in innovation that is the reason we ought to run parallel with it, to be capable as much as we can get advantage from these enhancements.

In the proposed technique the idea of evoting application is made utilizing android. Once the confirmation is done the voter is made to continue with the voting procedure. The primary motivation behind executing this idea is to build the voting rate. So that the voter is not required to visit the voting focus to make their choice and furthermore to maintain a strategic distance from fake voting.

\section{RELATED WORK}

1.1 FengYumeng,TianLiye,LiuFanbao,Gan Chong in 2012 proposed the looks into on the electronic voting plans toward reliable voting framework and examine issues around a few critical or dumbfounding properties. Electronic voting is a colossal field that contains a few procedures required with their points of interest and disadvantages. This examination region has been very beneficial, with various bearings investigated and captivating methods created. Electronic voting can possibly be the most solid, secure and reliable voting approach. Advanced innovation including mistake adjustment, 
vigorous capacity and cryptographic security offers the likelihood to record, transmit, store and check votes significantly more dependably than paper. This examination territory has been very profitable, with various bearings investigated and interesting procedures created. In this paper, we survey the investigates on the electronic voting plans toward reliable voting framework and examine the issues around a few vital properties and difficulties.

2.2Ari Juels ,Dario Catalano, Markus Jakobsson in 2005 We present a model for electronic race conspires that includes a more capable foe than past work. In specific, we permit the enemy to request of constrained voters that they vote in a specific way, refuse voting, or even uncover their mystery keys. We characterize a plan to be pressure safe on the off chance that it is infeasible for the foe to figure out whether a constrained voter consents to the requests. A first commitment of this paper is to depict and describe another and fortified foe for intimidation in decisions. (In doing as such, we furthermore show what we accept to be the main formal security definitions for electronic decisions of any sort.) A moment commitment is to illustrate a convention that is secure against this foe. While obviously a fortifying of assault models is of hypothetical significance, take note of that our outcomes lie near common sense. This is genuine both in that we display genuine dangers, (for example, vote-purchasing and votescratching off), also, in that our proposed convention joins a reasonable degree of effectiveness with a bizarre absence of auxiliary unpredictability. Moreover, past plans have required utilization of an untappable channel all through. Our own exclusive conveys the much more commonsense prerequisite of an unknown channel amid the throwing of tallies, and an untappable channel amid enlistment.

\subsection{Russell A. Fink, Alan T. Sherman, and} Richard Carback in 2009 We diminish the required trusted registering base for coordinate recording electronic (DRE) voting machines with a plan in light of confided in stage modules (TPMs). Our approach guarantees decision information respectability by restricting the voter's decisions with the displayed poll utilizing a stage vote (PVB) signature key oversaw by the TPM. The TPM can utilize the PVB key just when static estimations of the product mirror an uncompromised state and at the point when a region judge enters an uncommon secret key uncovered on decision day. Utilizing the PVB with the TPM can uncover approved programming, poll changes, vote altering, and production of fake decision records right on time in the race procedure. Our convention places confide in alter safe equipment, not in changeable framework programming [6]. In spite of the fact that we are not the first to recommend utilizing TPMs in voting, we are the first to give a nitty gritty building convention that ties the voter decisions with the exhibited tally and uses the TPM to authorize race approach. We show the convention, engineering, presumptions, what's more, security contentions in enough detail to bolster advance investigation or execution.

2.4. David Chaum, Richard T. Carback, Jeremy Clark, Aleksander Essex, Stefan Popoveniuc, Ronald L. Rivest, Peter Y. A. 
Ryan, Emily Shen, Alan T. Sherman, and Poorvi L. Vora in 2009. It permits voters to check race respectability from their determinations on the vote the distance to the last counts-by taking note of codes and checking for them on the web. Voters check Scantegrity II polls similarly as with routine optical output, however utilizing an uncommon vote stamping pen. Denoting a determination with this pen makes decipherable a generally imperceptible preprinted affirmation code. Affirmation codes are autonomous and arbitrary for each potential choice on each ticket. To confirm that their person votes are recorded accurately, voters can look into their ticket serial numbers on the web and check that their affirmation codes are posted accurately. The affirmation codes don't permit voters to demonstrate how they voted Be that as it may; the affirmation codes constitute persuading proof of blunder or impropriety if erroneous codes are posted on the web. Rightness of the last count as for the distributed codes is demonstrated by race authorities in a way that can be confirmed by any invested individual. In this way, trade off of either poll chain of authority or the product frameworks can't imperceptibly influence decision uprightness. Scantegrity II has beenexecuted and tried in little races in which tickets were checked either at the surveying place or halfway. Arrangements for its utilization in an open part decision have initiated.

2.5. Lucie Langer, Axel Schmidt, Johannes Buchmann,MelanieVolkamer, Alexander Stolfik in 2010,Proposed various levels of race mystery and undeniable nature. We likewise explore whether there exists a request for the diverse levels and give enemy models. The subsequent accumulation is valuable since it may not be fundamental, or even conceivable, to accomplish the most extreme level for all the security prerequisites in parallel. Hence, fitting levels of the necessities can be chosen for various sorts of decisions, e.g. parliamentary races versus decisions in affiliations. Electronic voting plans are relied upon to meet the fundamental security necessities for electronic voting. Be that as it may, altogether different feelings about these necessities exist in the e-voting group. This is because of the way that the security prerequisites include distinctive levels at which they can be met. For instance, widespread unquestionable status may, or may, exclude confirming qualification of the voters who took part in the race.

\section{PROPOSED SYSTEM}

Our android application has a web benefit call empowered just to the accessible pooling city, state or nation on that specific date and to those specific working hours. The voter can make just a single choice after that the session get shut instantly, and he can't ready to cast utilizing any of the confirmation, for example, Aadhar card or voter id. The tallying procedure and checking procedure are overseen by the manager; the numbering is done subsequent to making of choice has been done inside 60 minutes. The framework is more proficient and will have all sort of security system, for example, cryptography, and so on.

\section{ADVANTAGES}

The android application the client can send his vote from wherever. More secure and powerful application.Eliminates the fake voting. Every one of the residents can successfully take part in the race. this application working on 
10am to $6 \mathrm{pm}$. our system reduces the time for user.

\section{SYSTEM IMPLEMENTATION}

\section{System Design}

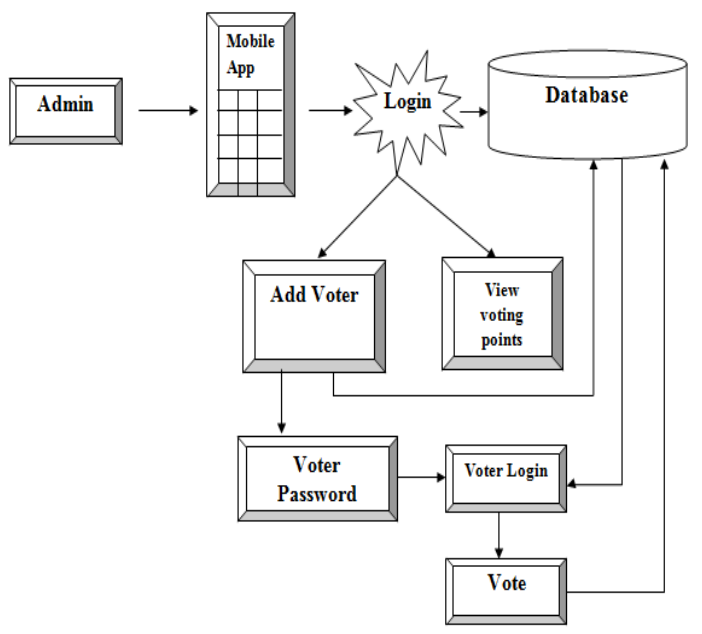

Figure 3.1Architecture Diagram

The figure 3.1 shows architecture of the proposed work, It uses android as Front End and android sqliteas Back End.

This process can be done through the following modules.
* User login
* User Voting page
* Admin login
* Add new voters
* View voters details
* Counting votes

\section{User Login}

In this module, the voter can login by the utilization of city code and voter ID for a specific time. In the event that the voter can as of now vote, then it is impractical to vote once more. For a specific time no one but you can login and vote .client can get the secret word from administrator when an administrator can include the client.

\section{User Voting Page}

In this module, the voter can see the images and voter can vote in this page. On the off chance that the voter can as of now vote, then it is unrealistic to vote once more. It advise that "Officially noted".

\section{Admin Login}

In this administrator can login by utilizing username and secret word.

\section{Authentication Module}

This module speaks to the validation, which is utilized for administrator and voter to login their subtle elements for the procedures. Verification is utilized as the premise or approval figuring out if a benefit will be conceded to a specific client or process. The approval procedures are done on the web server.

\section{Add New Voters}

In this module, the administrator can include the new voter's points of interest like city code, voter Id, aadhar card number, voter subtle elements and portable number.

\section{View Voter Details}

In this module, the administrator can see the voter points of interest like city code, voter Id, voter subtle elements.

\section{Counting Votes}

In this module, administrators check the quantity of votes and who wins the vote specifically zone in light of city code.

\section{Web Service Client Module}

This module has the way toward putting away the chose applicant data from the customer, which are send through the web benefit. All these data's will be put away in the database. Administrator keeping up a brought 
together server with a specific end goal to get the chose voter list from the database through web. In this module the competitor see they information recovered from the database. The Voter will utilize this rundown to play out the voting.

\section{CONCLUSION}

This Online Voting framework will deal with the Voter's data by which voter can login and utilize his voting rights. The framework will join all components of voting framework. It gives the devices to keeping up voter's vote to each gathering and it tally add up to number of votes of each gathering. There is a database which is kept up by the election commission of India in which every one of the names of voter with finish data is put away.

By web based voting framework rate of voting is increments. It diminishes the cost and time of voting procedure. It is anything but difficult to utilize and it is shift less tedious. It is anything but difficult to investigate

\section{FUTURE ENHANCEMENT}

The future extent of the venture incorporates the change in the security level of the entire framework. Notwithstanding that it is important to meet some other private primitives to enhance the security level of internet voting framework. The future change should likewise be possible for voice check, Fingerprint, so that the voters can vote with more confirmation.

\section{REFERENCES}

[1]FengYumeng,TianLiye,LiuFanbao,Gan Chong, "Electronic Voting: A Review and Taxonomy," 2012 International Conference on Industrial Control and Electronics Engineering, pp. 912-917, Aug. 2012.
[2] Ari Juels ,Dario Catalano, Markus Jakobsson, "Coercion-resistant electronic elections," in Towards Trustworthy Elections: New Directions in Electronic Voting, ser. LNCS. Springer, Feb. 2010, vol. 6000, pp. 3763.

[3] Russell A. Fink, Alan T. Sherman, and Richard Carback "TPM meets DRE: Reducing the trust base for electronic voting using trusted platform modules," IEEE Transactions on Information Forensics and Security, vol. 4, no. 4, pp. 628-637, Dec. 2009.

[4]David Chaum, Richard T. Carback, Jeremy Clark, Aleksander Essex, Stefan Popoveniuc, Ronald L. Rivest, Peter Y. A. Ryan, Emily Shen, Alan T. Sherman, and Poorvi L. VoraII: End-to-End verifiability by voter of optical scan elections through confirmation codes," IEEE Transactions on Information Forensics and Security, vol. 4, no. 4, pp. 611-627, Dec. 2009.

[5] Lucie Langer, Axel Schmidt, Johannes Buchmann,MelanieVolkamer, Alexander Stolfik"Towards a Framework on the Security Requirements for Electronic Voting Protocols," 2010

[6] Hyoseok Yoon, Nohyoung Park, Wonwoo Lee, Youngkyoon Jang and Woontack Woo, "QR Code Data Representation for Mobile Augmented Reality" International AR Standards Meeting- vol. 1, no.212, pp. 253-258, 2011.

[7] R. Ara'ujo, S. Foulle, and J. Traor'e, “A practical and secure coercion-resistant scheme for Internet voting," in Towards Trustworthy Elections: New Directions in Electronic Voting,ser. LNCS. Springer, Feb. 2010, vol. 6000, pp. 330-342. 
[8] D. Chaum, R. T. Carback, J. Clark, A. Essex, S. Popoveniuc, R. L. Rivest, P. Y. A. Ryan, E. Shen, A. T. Sherman, and P. L. Vora, "Scantegrity II: End-to-End verifiability by voters of optical scan elections through confirmation codes," IEEETransactions on Information Forensics and Security, vol. 4, no. 4, pp. 611-627, Dec. 2009.

[9] D. Chaum, A. Essex, R. Carback, J. Clark, S. Popoveniuc, A. T. Sherman, and P. Vora, "Scantegrity: End-to-end voter verifiable optical-scan voting," IEEE Security Privacy, vol. 6, no. 3, pp. 40-46, May/Jun. 2008.

[10] E. Hubbers, B. Jacobs, and W. Pieters, "RIES - Internet Voting in Action," in COMPSAC '05: Proceedings of the 29th Annual International Computer Software and Applications Conference. Washington, DC, USA: IEEE Computer Society,2005, pp. 417-424. 